\title{
ON 2-ORTHOGONAL POLYNOMIALS OF LAGUERRE TYPE
}

\section{KHALFA DOUAK}

(Received 7 July 1997 and in revised form 10 February 1998)

\begin{abstract}
Let $\left\{P_{n}\right\}_{n \geq 0}$ be a sequence of 2-orthogonal monic polynomials relative to linear functionals $\omega_{0}$ and $\omega_{1}$ (see Definition 1.1). Now, let $\left\{Q_{n}\right\}_{n \geq 0}$ be the sequence of polynomials defined by $Q_{n}:=(n+1)^{-1} P_{n+1}^{\prime}, n \geq 0$. When $\left\{Q_{n}\right\}_{n \geq 0}$ is, also, 2-orthogonal, $\left\{P_{n}\right\}_{n \geq 0}$ is called "classical" (in the sense of having the Hahn property). In this case, both $\left\{P_{n}\right\}_{n \geq 0}$ and $\left\{Q_{n}\right\}_{n \geq 0}$ satisfy a third-order recurrence relation (see below). Working on the recurrence coefficients, under certain assumptions and well-chosen parameters, a classical family of 2-orthogonal polynomials is presented. Their recurrence coefficients are explicitly determined. A generating function, a third-order differential equation, and a differentialrecurrence relation satisfied by these polynomials are obtained. We, also, give integral representations of the two corresponding linear functionals $\omega_{0}$ and $\omega_{1}$ and obtain their weight functions which satisfy a second-order differential equation. From all these properties, we show that the resulting polynomials are an extention of the classical Laguerre's polynomials and establish a connection between the two kinds of polynomials.
\end{abstract}

Keywords and phrases. Orthogonal polynomials, $d$-orthogonal polynomials, Laguerre polynomials, Sheffer polynomials, recurrence relations, integral representations.

1991 Mathematics Subject Classification. 33C45, 42C05.

1. Introduction. It is well known (see, e.g., [4]) that the generalized Laguerre polynomials $\left\{L_{n}^{(\alpha)}\right\}_{n \geq 0}$, for $\alpha>-1$, are orthogonal with respect to the weight function $\varrho(x)=x^{\alpha} e^{-x}$ on the interval $0 \leq x<+\infty$, that is,

$$
\int_{0}^{+\infty} L_{m}^{(\alpha)}(x) L_{n}^{(\alpha)}(x) x^{\alpha} e^{-x} d x=\frac{\Gamma(n+\alpha+1)}{n !} \delta_{m, n}, \quad m, n \geq 0
$$

They are defined by the generating function

$$
(1-t)^{-\alpha-1} e^{-x t /(1-t)}=\sum_{n=0}^{\infty} L_{n}^{(\alpha)}(x) t^{n}
$$

Their corresponding monic polynomials $\left\{\hat{L}_{n}^{(\alpha)}\right\}_{n \geq 0}$ are defined by $\hat{L}_{n}^{(\alpha)}=(-1)^{n} n ! L_{n}^{(\alpha)}$, $n \geq 0$ and satisfy the second-order recurrence relation

$$
\begin{gathered}
\hat{L}_{n+2}^{(\alpha)}(x)=(x-(2 n+\alpha+3)) \hat{L}_{n+1}^{(\alpha)}(x)-(n+1)(n+\alpha+1) \hat{L}_{n}^{(\alpha)}(x), \quad n \geq 0, \\
\hat{L}_{0}^{(\alpha)}(x)=1, \\
\hat{L}_{1}^{(\alpha)}(x)=x-(\alpha+1),
\end{gathered}
$$


as well as the following relations:

$$
\begin{aligned}
\frac{d}{d x} \hat{L}_{n+1}^{(\alpha)}(x) & =(n+1) \hat{L}_{n}^{(\alpha+1)}(x), & & n \geq 0, \\
\hat{L}_{n+1}^{(\alpha)}(x) & =\hat{L}_{n+1}^{(\alpha+1)}(x)+(n+1) \hat{L}_{n}^{(\alpha+1)}(x), & & n \geq 0, \\
x \hat{L}_{n}^{(\alpha+1)}(x) & =\hat{L}_{n+1}^{(\alpha)}(x)+(n+\alpha+1) \hat{L}_{n}^{(\alpha)}(x), & & n \geq 0 .
\end{aligned}
$$

Recently, within the framework of the $d$-orthogonality of polynomials or polynomials of simultaneous orthogonality studied in $[12,11,16]$ which does not really have the same orthogonality relations but are considered to be orthogonal relative to positive measures, new kinds of $d$-orthogonal polynomials have been the subject of various investigations $[1,3,5,9,15]$. In particular, those having some properties that are analogous to the classical orthogonal polynomials.

In this paper, when $d=2$, under special conditions and well-chosen parameters, we give a family of 2-orthogonal "classical" polynomials which are a natural extension of the classical Laguerre polynomials. These polynomials have some properties analogous to those satisfied by the classical Laguerre polynomials. Their recurrence coefficients and generating function are explicitly determined, a differential-recurrence relation and a third-order differential equation are obtained. We denote these polynomials by $P_{n}(\cdot ; \alpha)$, where $\alpha$ is an arbitrary parameter. They are called the 2-orthogonal polynomials of Laguerre type related to the two linear functionals $\omega_{0}, \omega_{1}$, where $\omega_{0}$ satisfies a second-order differential (distributional) equation and $\omega_{1}$ is given in terms of $\omega_{0}$ and $\omega_{0}^{\prime}$ (see equations (4.13), (4.14)). Finally, one of the problems is to determine integral representations of both functionals $\omega_{0}$ and $\omega_{1}$. Indeed, applying the method explained in [8], if we denote by $\mathscr{W}_{0}$ (resp., $\mathscr{W}_{1}$ ) the weight function representing the functional $\omega_{0}$ (resp., $\omega_{1}$ ), we obtain that when $\alpha>-1, \mathcal{W}_{0}(x)=e^{-1} \varrho(x) I_{\alpha}^{*}(x)$ on the interval $0 \leq x<+\infty$, with $\varrho(x)=x^{\alpha} e^{-x}$ being the weight function related to the classical Laguerre polynomials, and $I_{\alpha}^{*}$ an entire function defined by $I_{\alpha}^{*}(x)=x^{-\alpha / 2} I_{\alpha}(2 \sqrt{x})$, where $I_{\alpha}$ is the modified Bessel function of the first kind.

Let us now recall some results which we need below. Let $\left\{P_{n}\right\}_{n \geq 0}$ be a sequence of monic polynomials and $\left\{\omega_{n}\right\}_{n \geq 0}$ be its dual sequence defined by $\left\langle\omega_{m}, P_{n}\right\rangle=\delta_{m, n}$, $m, n \geq 0$, where $\langle$,$\rangle is the duality brackets between \mathscr{P}$ (the vector space of polynomials with coefficients in $\mathbb{C}$ ) and its dual $\mathscr{P}^{\prime}$.

LEMMA 1.1 [14]. For any linear functional $u$ and integer $p \geq 1$, the following two statements are equivalent:

(i) $\left\langle u, P_{p-1}\right\rangle \neq 0 ;\left\langle u, P_{n}\right\rangle=0, n \geq p$;

(ii) $\exists \lambda_{v} \in \mathbb{C}, 0 \leq v \leq p-1, \lambda_{p-1} \neq 0$ such that $u=\sum_{v=0}^{p-1} \lambda_{v} u_{v}$.

DEFINITION 1.1. The sequence $\left\{P_{n}\right\}_{n \geq 0}$ is said to be $d$-orthogonal polynomials sequence ( $d$-OPS) with respect to the $d$-dimensional functional $\Omega={ }^{t}\left(\omega_{0}, \ldots, \omega_{d-1}\right)$ if it fulfills $[14,17]$

$$
\begin{aligned}
\left\langle\omega_{v}, P_{m} P_{n}\right\rangle & =0, \quad n \geq m d+v+1, \\
\left\langle\omega_{v}, P_{m} P_{m d+v}\right\rangle & \neq 0,
\end{aligned}
$$

for each integer $v$ with $v=0,1, \ldots, d-1$ and $m \geq 0$. 


\section{REMARK 1.1.}

(a) When $d=1$, we meet again the ordinary orthogonality.

(b) The linear functionals $\omega_{v}$ are not necessarily positive definite and the $d$-dimensional functional $\Omega$ is not unique. Indeed, according to Lemma 1.1, if $\left\{P_{n}\right\}_{n \geq 0}$ is $d$ orthogonal relative to the functional $u={ }^{t}\left(u_{1}, \ldots, u_{d}\right)$, we have

$$
\begin{aligned}
u_{\kappa}=\sum_{\nu=0}^{\kappa-1} \lambda_{v}^{\kappa} \omega_{\nu}, \quad \lambda_{\kappa-1}^{\kappa} \neq 0, & 1 \leq \kappa \leq d \\
& \Longleftrightarrow \omega_{v}=\sum_{k=1}^{v+1} \tau_{k}^{v} u_{k}, \quad \tau_{v+1}^{v} \neq 0,0 \leq v \leq d-1 .
\end{aligned}
$$

Thus, this notion of $d$-orthogonality for polynomials, defined and studied in a different context in [17], appears as a particular case of the general notion of biorthogonality described in [2]. A remarkable characterization of the $d$-orthogonal polynomials is that they satisfy a standard $(d+1)$-order recurrence relation, that is, a relation between $d+2$ consecutive polynomials [17]. Here, we work only with the canonical $d$-dimensional functional $\Omega={ }^{t}\left(\omega_{0}, \ldots, \omega_{d-1}\right)$ and in all the sequel we only consider the case $d=2$, that is, $\left\{P_{n}\right\}_{n \geq 0}$ is 2-OPS with respect to the linear functionals $\omega_{0}$ and $\omega_{1}$. In this case, the orthogonality relations are

$$
\begin{aligned}
\left\langle\omega_{0}, P_{m} P_{n}\right\rangle & =0, \quad n \geq 2 m+1 ; \\
\left\langle\omega_{0}, P_{m} P_{2 m}\right\rangle \neq 0, & m \geq 0
\end{aligned}
$$

and

$$
\begin{aligned}
\left\langle\omega_{1}, P_{m} P_{n}\right\rangle=0, & n \geq 2 m+2 ; \\
\left\langle\omega_{1}, P_{m} P_{2 m+1}\right\rangle \neq 0, & m \geq 0 .
\end{aligned}
$$

Then $\left\{P_{n}\right\}_{n \geq 0}$ satisfies a third-order recurrence relation $[14,17]$ which we write in the form

$$
\begin{aligned}
P_{n+3}(x) & =\left(x-\beta_{n+2}\right) P_{n+2}(x)-\alpha_{n+2} P_{n+1}(x)-\gamma_{n+1} P_{n}(x), \quad n \geq 0, \\
P_{0}(x) & =1, \quad P_{1}(x)=x-\beta_{0}, \quad P_{2}(x)=\left(x-\beta_{1}\right) P_{1}(x)-\alpha_{1}, \\
\gamma_{n+1} & \neq 0, \quad n \geq 0 \quad \text { (regularity conditions). }
\end{aligned}
$$

Let us now introduce the sequence of monic polynomials $\left\{Q_{n}:=(n+1)^{-1} P_{n+1}^{\prime}\right\}_{n \geq 0}$. We denote by $\left\{\tilde{\omega}_{n}\right\}_{n \geq 0}$ the dual sequence of $\left\{Q_{n}\right\}_{n \geq 0}$. According to the Hahn's property [10], if the sequence $\left\{Q_{n}\right\}_{n \geq 0}$ is also 2-orthogonal, then $\left\{P_{n}\right\}_{n \geq 0}$ is called a "classical" 2OPS. In this case, the sequence $\left\{Q_{n}\right\}_{n \geq 0}$, too, satisfies a third-order recurrence relation

$$
\begin{aligned}
Q_{n+3}(x) & =\left(x-\tilde{\beta}_{n+2}\right) Q_{n+2}(x)-\tilde{\alpha}_{n+2} Q_{n+1}(x)-\tilde{\gamma}_{n+1} Q_{n}(x), \quad n \geq 0, \\
Q_{0}(x) & =1, \quad Q_{1}(x)=x-\tilde{\beta}_{0}, \quad Q_{2}(x)=\left(x-\tilde{\beta}_{1}\right) Q_{1}(x)-\tilde{\alpha}_{1}, \\
\tilde{\gamma}_{n+1} & \neq 0, \quad n>0 .
\end{aligned}
$$

By differentiating (1.11) and using (1.12), we easily obtain

$$
\begin{aligned}
P_{n+3}(x)= & Q_{n+3}(x)+(n+3)\left(\beta_{n+3}-\tilde{\beta}_{n+2}\right) Q_{n+2}(x) \\
& +\left((n+2) \alpha_{n+3}-(n+3) \tilde{\alpha}_{n+2}\right) Q_{n+1}(x) \\
& +\left((n+1) \gamma_{n+2}-(n+3) \tilde{\gamma}_{n+1}\right) Q_{n}(x), \quad n \geq 0,
\end{aligned}
$$


with the initial conditions

$$
\begin{aligned}
& P_{0}(x)=Q_{0}(x)=1, \\
& P_{1}(x)=Q_{1}(x)+\beta_{1}-\tilde{\beta}_{0}, \\
& P_{2}(x)=Q_{2}(x)+2\left(\beta_{2}-\tilde{\beta}_{1}\right) Q_{1}(x)+\left(\alpha_{2}-\tilde{\alpha}_{1}\right) .
\end{aligned}
$$

Otherwise, when the 2-OPS $\left\{P_{n}\right\}_{n \geq 0}$ is "classical", it was obtained in [6] that the recurrence coefficients $\left\{\beta_{n}\right\},\left\{\tilde{\beta}_{n}\right\},\left\{\gamma_{n}^{\nu}\right\}$, and $\left\{\tilde{\gamma}_{n}^{v}\right\}(\nu=0,1)$ satisfy the following non-linear system which is valid for $n \geq 1$ :

$$
\begin{aligned}
(n+2) \tilde{\beta}_{n}-n \tilde{\beta}_{n-1}= & (n+1) \beta_{n+1}-(n-1) \beta_{n} ; \\
2 \tilde{\beta}_{0}= & \beta_{0}+\beta_{1}, \\
(n+3) \tilde{\alpha}_{n+1}-(n+1) \tilde{\alpha}_{n}= & (n+1) \alpha_{n+2}-(n-1) \alpha_{n+1} \\
& +(n+1)\left(\beta_{n+1}-\tilde{\beta}_{n}\right)^{2} ; \\
3 \tilde{\alpha}_{1}= & \alpha_{2}+\alpha_{1}+\left(\beta_{1}-\tilde{\beta}_{0}\right)^{2}, \\
(n+4) \tilde{\gamma}_{n+1}-(n+2) \tilde{\gamma}_{n}= & (n+1) \gamma_{n+2}-(n-1) \gamma_{n+1} \\
& +(n+1) \alpha_{n+2}\left(\beta_{n+2}+\beta_{n+1}-2 \tilde{\beta}_{n}\right) \\
& -(n+2) \tilde{\alpha}_{n+1}\left(2 \beta_{n+2}-\tilde{\beta}_{n+1}-\tilde{\beta}_{n}\right) ; \\
4 \tilde{\gamma}_{1}= & \gamma_{2}+\gamma_{1}+\alpha_{2}\left(\beta_{2}+\beta_{1}-2 \tilde{\beta}_{0}\right) \\
& -2 \tilde{\alpha}_{1}\left(2 \beta_{2}-\tilde{\beta}_{1}-\tilde{\beta}_{0}\right), \\
n \alpha_{n+1} \alpha_{n+2}+(n+2) \tilde{\alpha}_{n} \tilde{\alpha}_{n+1}- & 2(n+1) \tilde{\alpha}_{n} \alpha_{n+2} \\
= & (n+2) \tilde{\gamma}_{n}\left(2 \beta_{n+2}-\tilde{\beta}_{n+1}-\tilde{\beta}_{n-1}\right) \\
& -n \gamma_{n+1}\left(\beta_{n+2}+\beta_{n}-2 \tilde{\beta}_{n-1}\right) ; \\
n \alpha_{n+1} \gamma_{n+2}+n \gamma_{n+1} \alpha_{n+3}= & \tilde{\gamma}_{n}\left(2(n+2) \alpha_{n+3}-(n+3) \tilde{\alpha}_{n+2}\right) \\
& +\tilde{\alpha}_{n}\left(2(n+1) \gamma_{n+2}-(n+3) \tilde{\gamma}_{n+1}\right), \\
n \gamma_{n+1} \gamma_{n+3}= & \tilde{\gamma}_{n}\left(2(n+2) \gamma_{n+3}-(n+4) \tilde{\gamma}_{n+2}\right) .
\end{aligned}
$$

To solve this system, we pose

$$
\begin{array}{llll}
\tilde{\beta}_{n}=\beta_{n+1}+\delta_{n}, & n \geq 0, & \\
\tilde{\alpha}_{n}=\alpha_{n+1} \frac{n}{n+1} \rho_{n}, & n \geq 1 & \left(\rho_{n} \neq 0\right), \\
\tilde{\gamma}_{n}=\gamma_{n+1} \frac{n}{n+2} \theta_{n}, & n \geq 1 & \left(\theta_{n} \neq 0\right) .
\end{array}
$$

In its full generality, the above system has remained unsolved. However, if we impose symmetry [6] or are interested in particular conditions [5, 9, 15, 8], some solutions have been obtained. For instance, under the assumption $\left(\delta_{n}=0, n \geq 0\right.$, then $\beta_{n}=$ $\tilde{\beta}_{n}=$ const.), the system was solved in [6] and a class of "classical" 2-orthogonal polynomials were obtained. In this paper, we give another particular solution when $\delta_{n} \neq 0, n \geq 0$. 
2. Another particular solution of the system (1.15)-(1.23). First, under the transformations (1.24)-(1.26), the relation (1.13) becomes

$$
\begin{aligned}
P_{n+3}(x)= & Q_{n+3}(x)-(n+3) \delta_{n+2} Q_{n+2}(x) \\
& +(n+2) \alpha_{n+3}\left(1-\rho_{n+2}\right) Q_{n+1}(x) \\
& +(n+1) \gamma_{n+2}\left(1-\theta_{n+1}\right) Q_{n}(x), \quad n \geq 0,
\end{aligned}
$$

with

$$
\begin{aligned}
& P_{0}(x)=Q_{0}(x)=1, \\
& P_{1}(x)=Q_{1}(x)-\delta_{0}, \\
& P_{2}(x)=Q_{2}(x)-2 \delta_{1} Q_{1}(x)+\alpha_{2}\left(1-\rho_{1}\right) .
\end{aligned}
$$

Likewise, by using (1.24)-(1.26), the system (1.15)-(1.23) is, also, transformed to

$$
\begin{aligned}
& \beta_{n+1}-\beta_{n}=n \delta_{n-1}-(n+2) \delta_{n}, \quad n \geq 1, \\
& \beta_{1}-\beta_{0}=-2 \delta_{0}, \\
& {\left[(n+3) \rho_{n+1}-(n+2)\right] \frac{\alpha_{n+2}}{n+2}-\left[n \rho_{n}-(n-1)\right] \frac{\alpha_{n+1}}{n+1}=\delta_{n}^{2}, \quad n \geq 1,} \\
& \left(3 \rho_{1}-2\right) \frac{\alpha_{2}}{2}-\alpha_{1}=\delta_{0}^{2}, \\
& {\left[(n+4) \theta_{n+1}-(n+3)\right] \frac{\gamma_{n+2}}{n+3}-\left[n \theta_{n}-(n-1)\right] \frac{\gamma_{n+1}}{n+1}} \\
& \quad=\alpha_{n+2}\left\{\left(\beta_{n+2}-\beta_{n+1}-2 \delta_{n}\right)-\rho_{n+1}\left(\beta_{n+2}-\beta_{n+1}-\delta_{n+1}-\delta_{n}\right)\right\}, \quad n \geq 1, \\
& \left(4 \theta_{1}-3\right) \frac{\gamma_{2}}{3}-\gamma_{1}=\alpha_{2}\left\{\left(\beta_{2}-\beta_{1}-2 \delta_{0}\right)-\rho_{1}\left(\beta_{2}-\beta_{1}-\delta_{1}-\delta_{0}\right)\right\}, \quad n \geq 1, \\
& \left(2 \rho_{n}-\rho_{n+1} \rho_{n}-1\right) \alpha_{n+1} \alpha_{n+2} \\
& \quad=\gamma_{n+1}\left\{\left(\beta_{n+2}-\beta_{n}-\delta_{n+1}-\delta_{n-1}\right)-\theta_{n}\left(\beta_{n+2}-\beta_{n}-2 \delta_{n-1}\right)\right\}, \quad n \geq 1, \\
& \alpha_{n+1} \gamma_{n+2}\left[2 \rho_{n}-\rho_{n} \theta_{n+1}-1\right]+\alpha_{n+3} \gamma_{n+1}\left[2 \theta_{n}-\theta_{n} \rho_{n+2}-1\right]=0, \quad n \geq 1, \\
& \theta_{n+2}+\frac{1}{\theta_{n}}=2, \quad n \geq 1 .
\end{aligned}
$$

As the starting point for the solution of the above system, we begin with equation (2.11) which is Riccati equation and for which $\theta_{n}=1, n \geq 1$ is a trivial solution. In that case, the above system becomes

$$
\begin{aligned}
& \beta_{n+1}-\beta_{n}=n \delta_{n-1}-(n+2) \delta_{n}, \quad n \geq 1 ; \\
& \beta_{1}-\beta_{0}=-2 \delta_{0}, \\
& {\left[(n+3) \rho_{n+1}-(n+2)\right] \frac{\alpha_{n+2}}{n+2}-\left[n \rho_{n}-(n-1)\right] \frac{\alpha_{n+1}}{n+1}=\delta_{n}^{2}, \quad n \geq 1 ;} \\
& \left(3 \rho_{1}-2\right) \frac{\alpha_{2}}{2}-\alpha_{1}=\delta_{0}^{2},
\end{aligned}
$$




$$
\begin{aligned}
& \frac{\gamma_{n+2}}{n+3}-\frac{\gamma_{n+1}}{n+1} \\
& \quad=\alpha_{n+2}\left\{\left(\beta_{n+2}-\beta_{n+1}-2 \delta_{n}\right)-\rho_{n+1}\left(\beta_{n+2}-\beta_{n+1}-\delta_{n+1}-\delta_{n}\right)\right\}, \quad n \geq 1 ; \\
& \frac{\gamma_{2}}{3}-\gamma_{1}=\alpha_{2}\left\{\left(\beta_{2}-\beta_{1}-2 \delta_{0}\right)-\rho_{1}\left(\beta_{2}-\beta_{1}-\delta_{1}-\delta_{0}\right)\right\}, \\
& {\left[2 \rho_{n}-\rho_{n+1} \rho_{n}-1\right] \alpha_{n+1} \alpha_{n+2}=-\gamma_{n+1}\left(\delta_{n+1}-\delta_{n-1}\right), \quad n \geq 1,} \\
& \alpha_{n+1} \gamma_{n+2}\left[\rho_{n}-1\right]-\alpha_{n+3} \gamma_{n+1}\left[\rho_{n+2}-1\right]=0, \quad n \geq 1 .
\end{aligned}
$$

It may be seen that equation (2.19) is verified if $\rho_{n}=1, n \geq 1$. Thus, in view of this remark, the two following cases may be distinguished:

$$
\rho_{n}=1, \quad n \geq 1 \text { and } \rho_{n} \neq 1, \quad n \geq 1 \text {. }
$$

In this paper, we consider only the first case, that is, $\rho_{n}=1, n \geq 1$ (the simplest case). Hence, the system (2.12)-(2.19) becomes

$$
\begin{aligned}
\beta_{n+1}-\beta_{n} & =n \delta_{n-1}-(n+2) \delta_{n}, \quad n \geq 0,\left(\delta_{-1}=0\right), \\
\frac{\alpha_{n+2}}{n+2}-\frac{\alpha_{n+1}}{n+1} & =\delta_{n}^{2}, \quad n \geq 0, \\
\frac{\gamma_{n+2}}{n+3}-\frac{\gamma_{n+1}}{n+1} & =\alpha_{n+2}\left(\delta_{n+1}-\delta_{n}\right), \quad n \geq 0, \\
\gamma_{n+2}\left(\delta_{n+2}-\delta_{n}\right) & =0, \quad n \geq 0 .
\end{aligned}
$$

Since $\gamma_{n+1} \neq 0$, shifting $n \longrightarrow n+1$, equation (2.24) leads to $\delta_{n+2}-\delta_{n}=0, n \geq 0$, whose solutions are given in the following

$$
\begin{aligned}
\delta_{2 n} & =\delta_{0}, & & n \geq 0, \\
\delta_{2 n+1} & =\delta_{1}, & & n \geq 0,
\end{aligned}
$$

with $\delta_{0}=\left(\beta_{0}-\beta_{1}\right) / 2$ and $\delta_{1}=\left(\beta_{0}+\beta_{1}-2 \beta_{2}\right) / 6$. It may be seen that $\delta_{1}=\delta_{0}$ if and only if $\beta_{2}=2 \beta_{1}-\beta_{0}$. Thus, two cases arise.

(A) $\delta_{1}=\delta_{0}$,

(B) $\delta_{1} \neq \delta_{0}$.

2.1. The case (A) $\left(\delta_{1}=\delta_{0}\right)$. In this case, the system (2.21)-(2.24) leads to

$$
\begin{aligned}
\beta_{n+1}-\beta_{n} & =-2 \delta_{0}, \quad n \geq 0, \\
\frac{\alpha_{n+2}}{n+2}-\frac{\alpha_{n+1}}{n+1} & =\delta_{0}^{2}, \quad n \geq 0, \\
\frac{\gamma_{n+2}}{n+3}-\frac{\gamma_{n+1}}{n+1} & =0, \quad n \geq 0 .
\end{aligned}
$$

From these, we easily obtain

$$
\begin{aligned}
\beta_{n} & =\beta_{0}-2 \delta_{0} n, \quad n \geq 0, \\
\alpha_{n+1} & =(n+1)\left(\delta_{0}^{2} n+\alpha_{1}\right), \quad n \geq 0, \\
\gamma_{n+1} & =\frac{1}{2} \gamma_{1}(n+2)(n+1), \quad n \geq 0,
\end{aligned}
$$


and

$$
\begin{aligned}
\tilde{\beta}_{n} & =\beta_{0}-\delta_{0}(2 n+1), \quad n \geq 0, \\
\tilde{\alpha}_{n+1} & =(n+1)\left(\delta_{0}^{2}(n+1)+\alpha_{1}\right), \quad n \geq 0, \\
\tilde{\gamma}_{n+1} & =\frac{1}{2} \gamma_{1}(n+2)(n+1), \quad n \geq 0 .
\end{aligned}
$$

Note that if $\delta_{0}=0$, we obtain $\tilde{\beta}_{n}=\beta_{n}, \tilde{\alpha}_{n}=\alpha_{n}$ and $\tilde{\gamma}_{n}=\gamma_{n}$. Then $P_{n}=Q_{n}, n=$ $0,1,2, \ldots$ and $\left\{P_{n}\right\}_{n \geq 0}$ is, at the same time, 2-orthogonal and Appell sequence [5, 6]. In this case, the polynomials obtained are the analogous of the classical orthogonal polynomials of Hermite.

2.2. The case (B) $\left(\delta_{1} \neq \delta_{0}\right)$. First, from (2.21), we obtain, for $n \rightarrow 2 n+1$ and $n \rightarrow$ $2 n, \beta_{2 n}=\beta_{0}-\left(\delta_{0}+3 \delta_{1}\right) n, n \geq 0$ and $\beta_{2 n+1}=\beta_{1}-\left(\delta_{1}+3 \delta_{0}\right) n, n \geq 0$, respectively.

Secondly, from (2.22), for $n \longrightarrow 2 n$ and $n \longrightarrow 2 n+1$ we, respectively, obtain

$$
\begin{array}{ll}
\frac{\alpha_{2 n+2}}{2 n+2}-\frac{\alpha_{2 n+1}}{2 n+1}=\delta_{0}^{2}, & n \geq 0, \\
\frac{\alpha_{2 n+3}}{2 n+3}-\frac{\alpha_{2 n+2}}{2 n+2}=\delta_{1}^{2}, & n \geq 0,
\end{array}
$$

These give easily

$$
\begin{aligned}
& \alpha_{2 n+1}=(2 n+1)\left(\left(\delta_{0}^{2}+\delta_{1}^{2}\right) n+\alpha_{1}\right), \quad n \geq 0, \\
& \alpha_{2 n+2}=(2 n+2)\left(\left(\delta_{0}^{2}+\delta_{1}^{2}\right) n+\alpha_{1}+\delta_{0}^{2}\right), \quad n \geq 0 .
\end{aligned}
$$

Finally, from (2.23) and taking into account the last results, we, also, obtain

$$
\begin{array}{ll}
\frac{\gamma_{2 n+2}}{2 n+3}-\frac{\gamma_{2 n+1}}{2 n+1}=\left(\delta_{1}-\delta_{0}\right) \alpha_{2 n+2}, & n \geq 0, \\
\frac{\gamma_{2 n+3}}{2 n+4}-\frac{\gamma_{2 n+2}}{2 n+2}=\left(\delta_{0}-\delta_{1}\right) \alpha_{2 n+3}, & n \geq 0,
\end{array}
$$

which yield

$$
\begin{aligned}
& \gamma_{2 n+1}=(2 n+2)(2 n+1)\left(\left(\delta_{0}-\delta_{1}\right) \delta_{1}^{2} n+\frac{\gamma_{1}}{2}\right), \quad n \geq 0, \\
& \gamma_{2 n+2}=(2 n+3)(2 n+2)\left(\left(\delta_{1}-\delta_{0}\right) \delta_{0}^{2}(n+1)+\frac{\gamma_{1}}{2}+\left(\delta_{1}-\delta_{0}\right) \alpha_{1}\right), \quad n \geq 0 .
\end{aligned}
$$

Two subcases arise.

(B) $\delta_{0}=0$.

$$
\begin{aligned}
\beta_{2 n} & =\beta_{0}-3 \delta_{1} n, \quad n \geq 0 ; \\
\beta_{2 n+1} & =\beta_{1}-\delta_{1} n, \quad n \geq 0, \\
\alpha_{2 n+1} & =(2 n+1)\left(\delta_{1}^{2} n+\alpha_{1}\right), \quad n \geq 0 ; \\
\alpha_{2 n+2} & =(2 n+2)\left(\delta_{1}^{2} n+\alpha_{1}\right), \quad n \geq 0, \\
\gamma_{2 n+1} & =(2 n+2)(2 n+1)\left(-\delta_{1}^{3} n+\frac{\gamma_{1}}{2}\right), \quad n \geq 0, \\
\gamma_{2 n+2} & =(2 n+3)(2 n+2)\left(\delta_{1} \alpha_{1}+\frac{\gamma_{1}}{2}\right), \quad n \geq 0 .
\end{aligned}
$$


$\left(B_{2}\right) \delta_{1}=0$

$$
\begin{aligned}
\beta_{2 n} & =\beta_{0}-\delta_{0} n, \quad n \geq 0 ; \\
\beta_{2 n+1} & =\beta_{1}-3 \delta_{0} n, \quad n \geq 0, \\
\alpha_{2 n+1} & =(2 n+1)\left(\delta_{0}^{2} n+\alpha_{1}\right), \quad n \geq 0 ; \\
\alpha_{2 n+2} & =(2 n+2)\left(\delta_{0}^{2}(n+1)+\alpha_{1}\right), \quad n \geq 0, \\
\gamma_{2 n+1} & =\frac{\gamma_{1}}{2}(2 n+2)(2 n+1), \quad n \geq 0 ; \\
\gamma_{2 n+2} & =(2 n+3)(2 n+2)\left(-\delta_{0}^{3}(n+1)-\delta_{0} \alpha_{1}+\frac{\gamma_{1}}{2}\right), \quad n \geq 0 .
\end{aligned}
$$

From now on, we are only interested in the sequence of polynomials obtained in case (A) with $\delta_{0} \neq 0$ (fixed), and, under some conditions, we shall be concerned mainly with the properties of the resulting polynomials. We show that some of these properties are analogous to the Laguerre's polynomials ones. For this, we note first that when $\theta_{n}=1$ and $\rho_{n}=1$, the relations (2.1) and (2.2) become

$$
P_{n+1}(x)=Q_{n+1}(x)-(n+1) \delta_{n} Q_{n}(x), \quad n \geq 0 .
$$

This relation plays a fundamental role in the next section, it allows us to derive some properties as differential-recurrence relation, a third-order differential equation, and a generating function of the resulting polynomials.

\section{Some properties of the sequence obtained in case A}

\subsection{A differential-recurrence relation}

Proposition 3.1. The sequence $\left\{P_{n}\right\}_{n \geq 0}$ obtained in case (A) satisfies the following differential-recurrence relation:

$$
\phi(x) Q_{n}(x)=P_{n+1}(x)-a_{n} P_{n}(x)-b_{n} P_{n-1}(x), \quad n \geq 0\left(P_{-1}=0\right),
$$

where

$$
\begin{aligned}
\phi(x) & =x-\left(\beta_{0}+\frac{\alpha_{1}}{\delta_{0}}+\frac{\gamma_{1}}{2 \delta_{0}^{2}}\right), \\
a_{n} & =\delta_{0} n+\frac{\alpha_{1}}{\delta_{0}}+\frac{\gamma_{1}}{2 \delta_{0}^{2}}, \quad n \geq 0, \\
b_{n} & =\frac{\gamma_{1}}{2 \delta_{0}} n, \quad n \geq 0 .
\end{aligned}
$$

Proof. First, from (2.41), we have

$$
P_{n+2}^{\prime}(x)=(n+2) P_{n+1}(x)+\delta_{0}(n+2) P_{n+1}^{\prime}(x), \quad n \geq 0
$$

which we write for the subscripts $n, n+1$, and $n+3$ as follows:

$$
\begin{aligned}
P_{n}^{\prime}(x) & =n P_{n-1}(x)+\delta_{0} n P_{n-1}^{\prime}(x), \quad n \geq 0, \\
P_{n+1}^{\prime}(x) & =(n+1) P_{n}(x)+\delta_{0}(n+1) P_{n}^{\prime}(x), \quad n \geq 0, \\
P_{n+3}^{\prime}(x) & =(n+3) P_{n+2}(x)+\delta_{0}(n+3) P_{n+2}^{\prime}(x), \quad n \geq 0 .
\end{aligned}
$$


Substituting for $P_{n+2}^{\prime}$ from (3.5) into (3.8), we get

$$
\begin{aligned}
P_{n+3}^{\prime}(x)= & (n+3) P_{n+2}(x)+\delta_{0}(n+3)(n+2) P_{n+1}(x) \\
& +\delta_{0}^{2}(n+3)(n+2) P_{n+1}^{\prime}(x), \quad n \geq 0 .
\end{aligned}
$$

Now, according to (2.29), the recurrence relation (1.11) gives, for $n \geq 0$,

$$
\begin{aligned}
P_{n+3}(x)= & \left(x-\beta_{0}+2 \delta_{0}(n+2)\right) P_{n+2}(x) \\
& -(n+2)\left(\delta_{0}^{2}(n+1)+\alpha_{1}\right) P_{n+1}(x)-\frac{\gamma_{1}}{2}(n+2)(n+1) P_{n}(x),
\end{aligned}
$$

which gives by differentiation

$$
\begin{aligned}
P_{n+2}(x)= & P_{n+3}^{\prime}(x)-\left(x-\beta_{0}+2 \delta_{0}(n+2)\right) P_{n+2}^{\prime}(x) \\
& +(n+2)\left(\delta_{0}^{2}(n+1)+\alpha_{1}\right) P_{n+1}^{\prime}(x) \\
& +\frac{\gamma_{1}}{2}(n+2)(n+1) P_{n}^{\prime}(x), \quad n \geq 0 .
\end{aligned}
$$

Substituting for $P_{n+3}^{\prime}, P_{n+2}^{\prime}$, and $P_{n}^{\prime}$ from (3.9), (3.5), and (3.7), respectively, into (3.11) yields

$$
\begin{aligned}
\left(-\delta_{0}\left(x-\beta_{0}\right)\right. & \left.+\alpha_{1}+\frac{\gamma_{1}}{2 \delta_{0}}\right) P_{n+1}^{\prime}(x) \\
& =-P_{n+2}(x)+\left(x-\beta_{0}+\delta_{0}(n+1)\right) P_{n+1}(x)+\frac{\gamma_{1}}{2 \delta_{0}}(n+1) P_{n}(x) .
\end{aligned}
$$

From this, using the recurrence relation (3.10) by shifting $n \rightarrow n-1$, we easily obtain the differential relation (3.1).

\subsection{A third-order differential equation}

Proposition 3.2. The polynomials $P_{n}, n=0,1, \ldots$ satisfy the following third-order differential equation:

$$
\delta_{0}^{2} \phi(x) Y^{\prime \prime \prime}+\left(2 \delta_{0}\left(x-\beta_{0}\right)-\alpha_{1}+\delta_{0}^{2}\right) Y^{\prime \prime}+\left(x-\beta_{0}-\delta_{0}(n-1)\right) Y^{\prime}-n Y=0, \quad n \geq 0,
$$

where $Y=P_{n}(x)$.

Proof. From (3.1), we have

$$
\phi(x) P_{n+1}^{\prime}(x)=(n+1) P_{n+1}(x)-(n+1) a_{n} P_{n}(x)-(n+1) b_{n} P_{n-1}(x), \quad n \geq 0 .
$$

Differentiating twice, we successively obtain

$$
\phi(x) P_{n+1}^{\prime \prime}(x)=n P_{n+1}^{\prime}(x)-(n+1) a_{n} P_{n}^{\prime}(x)-(n+1) b_{n} P_{n-1}^{\prime}(x), \quad n \geq 0
$$

and

$$
\phi(x) P_{n+1}^{\prime \prime \prime}(x)=(n-1) P_{n+1}^{\prime \prime}(x)-(n+1) a_{n} P_{n}^{\prime \prime}(x)-(n+1) b_{n} P_{n-1}^{\prime \prime}(x), \quad n \geq 0 .
$$


Now, from the recurrence relation (3.10), by shifting $n \rightarrow n-1$, we get

$$
\begin{aligned}
\frac{\gamma_{1}}{2}(n+1) n P_{n-1}(x)= & \left(x-\beta_{0}+2(n+1) \delta_{0}\right) P_{n+1}(x) \\
& -(n+1)\left(n \delta_{0}^{2}+\alpha_{1}\right) P_{n}(x)-P_{n+2}(x),
\end{aligned}
$$

and by differentiation, we obtain

$$
\begin{aligned}
\frac{\gamma_{1}}{2}(n+1) n P_{n-1}^{\prime}(x)= & P_{n+1}(x)+\left(x-\beta_{0}+2 \delta_{0}(n+1)\right) P_{n+1}^{\prime}(x) \\
& -(n+1)\left(\delta_{0}^{2} n+\alpha_{1}\right) P_{n}^{\prime}(x)-P_{n+2}^{\prime}(x) .
\end{aligned}
$$

Using (3.5) to eliminate $P_{n+2}^{\prime}$, the last equation becomes

$$
\begin{aligned}
\frac{\gamma_{1}}{2}(n+1) n P_{n-1}^{\prime}(x)= & -(n+1) P_{n+1}(x)+\left(x-\beta_{0}+\delta_{0} n\right) P_{n+1}^{\prime}(x) \\
& -(n+1)\left(\delta_{0}^{2} n+\alpha_{1}\right) P_{n}^{\prime}(x)
\end{aligned}
$$

which yields by differentiating again

$$
\begin{aligned}
\frac{\gamma_{1}}{2}(n+1) n P_{n-1}^{\prime \prime}(x)= & -n P_{n+1}^{\prime}(x)+\left(x-\beta_{0}+\delta_{0} n\right) P_{n+1}^{\prime \prime}(x) \\
& -(n+1)\left(\delta_{0}^{2} n+\alpha_{1}\right) P_{n}^{\prime \prime}(x),
\end{aligned}
$$

and for $n \longrightarrow n+1$

$$
\begin{aligned}
\frac{\gamma_{1}}{2}(n+2)(n+1) P_{n}^{\prime \prime}(x)= & -(n+1) P_{n+2}^{\prime}(x)+\left(x-\beta_{0}+\delta_{0}(n+1)\right) P_{n+2}^{\prime \prime}(x) \\
& -(n+2)\left(\delta_{0}^{2}(n+1)+\alpha_{1}\right) P_{n+1}^{\prime \prime}(x) .
\end{aligned}
$$

Using (3.5) again, we obtain

$$
\frac{\gamma_{1}}{2}(n+1) P_{n}^{\prime \prime}(x)=\left(\delta_{0}\left(x-\beta_{0}\right)-\alpha_{1}\right) P_{n+1}^{\prime \prime}(x)+\left(x-\beta_{0}\right) P_{n+1}^{\prime}(x)-(n+1) P_{n+1}(x) .
$$

Then (3.20) becomes

$$
\begin{aligned}
\frac{\gamma_{1}}{2}(n+1) n P_{n-1}^{\prime \prime}(x)= & \frac{2}{\gamma_{1}}\left(\delta_{0}^{2} n+\alpha_{1}\right)(n+1) P_{n+1}(x) \\
& -\left(\frac{2}{\gamma_{1}}\left(\delta_{0}^{2} n+\alpha_{1}\right)\left(x-\beta_{0}\right)+n\right) P_{n+1}^{\prime}(x) \\
& +\left(x-\beta_{0}+\delta_{0} n-\frac{2}{\gamma_{1}}\left(\delta_{0}^{2} n+\alpha_{1}\right)\left(\delta_{0}\left(x-\beta_{0}\right)-\alpha_{1}\right)\right) P_{n+1}^{\prime \prime}(x) .
\end{aligned}
$$

Now, by using (3.22) and (3.23) to eliminate $P_{n}^{\prime \prime}$ and $P_{n-1}^{\prime \prime}$ in (3.16) and shifting $n \longrightarrow$ $n-1$, the differential equation (3.13) follows easily.

REMARK 3.1. (1) Since the values $\beta_{0}, \delta_{0}, \alpha_{1}$, and $\gamma_{1}$ are arbitrary, with $\delta_{0} \neq 0$ and $\gamma_{1} \neq 0$, then the polynomials obtained in case (A) constitute, a priori, a four-parameter 
family. But, in the rest of this paper, we fix $\delta_{0}, \gamma_{1}$ and give special importance to the case when $\delta_{0}=-1$ and $\gamma_{1}=2$. In this case, the differential equation (3.13) becomes

$$
\left(x-\beta_{0}+\alpha_{1}-1\right) Y^{\prime \prime \prime}+\left(-2 x+2 \beta_{0}-\alpha_{1}+1\right) Y^{\prime \prime}+\left(x-\beta_{0}-1+n\right) Y^{\prime}-n Y=0, \quad n \geq 0 .
$$

In order to determine the family of 2-OPS analogous to the classical Laguerre's one, we first transform the singularity of the above differential equation to the origin. Indeed, it is clear that equation (3.24) has a singularity at the point $x_{0}=\beta_{0}-\alpha_{1}+1$. Then by an appropriate change of variable, this singularity may be transformed to the origin, that is, $\beta_{0}-\alpha_{1}+1=0$, i.e., $\alpha_{1}=\beta_{0}+1$. In this case, we pose $\beta_{0}=\alpha+2$ so that $\alpha_{1}=\alpha+3$. Thus, the differential equation (3.24) takes the form

$$
x Y^{\prime \prime \prime}+(-2 x+\alpha+2) Y^{\prime \prime}+(x-\alpha-3+n) Y^{\prime}-n Y=0, \quad n \geq 0 .
$$

Recall that the classical Laguerre polynomials satisfy the following second-order differential equation:

$$
x y^{\prime \prime}+(-x+\alpha+1) y^{\prime}+n y=0, \quad n \geq 0 .
$$

(2) Further, in this case the third-order recurrence relations (1.11) and (1.12) become

$$
\begin{aligned}
P_{n+3}(x)= & (x-(2(n+3)+\alpha)) P_{n+2}(x)-(n+2)(n+\alpha+4) P_{n+1}(x) \\
& -(n+2)(n+1) P_{n}(x), \quad n \geq 0 \\
P_{0}(x)= & 1 \\
P_{1}(x)= & x-(\alpha+2) \\
P_{2}(x)= & x^{2}-2(\alpha+3) x+(\alpha+4)(\alpha+1)+1
\end{aligned}
$$

and

$$
\begin{aligned}
Q_{n+3}(x)= & (x-(2(n+3)+\alpha+1)) Q_{n+2}(x)-(n+2)(n+\alpha+5) Q_{n+1}(x) \\
& -(n+2)(n+1) Q_{n}(x), \quad n \geq 0 \\
Q_{0}(x)= & 1 \\
Q_{1}(x)= & x-(\alpha+3) \\
Q_{2}(x)= & x^{2}-2(\alpha+4) x+(\alpha+5)(\alpha+2)+1 .
\end{aligned}
$$

From these, by taking into account the dependence on the parameter $\alpha$ and putting $P_{n}(x)=P_{n}(x ; \alpha), n \geq 0$, it may be seen that $Q_{n}(x)=P_{n}(x ; \alpha+1), n \geq 0$, i.e.,

$$
\frac{d}{d x} P_{n+1}(x ; \alpha)=(n+1) P_{n}(x ; \alpha+1), \quad n \geq 0 .
$$

Further, the relations (2.41) and (3.1), also, become

$$
\begin{aligned}
P_{n+1}(x ; \alpha) & =P_{n+1}(x ; \alpha+1)+(n+1) P_{n}(x ; \alpha+1), \quad n \geq 0, \\
x P_{n}(x ; \alpha+1) & =P_{n+1}(x ; \alpha)+(n+\alpha+2) P_{n}(x ; \alpha)+n P_{n-1}(x ; \alpha), \quad n \geq 0 .
\end{aligned}
$$

The relations (3.29)-(3.31) are analogous to the relations (1.4)-(1.6) satisfied by classical Laguerre polynomials $\left\{L_{n}^{(\alpha)}\right\}_{n \geq 0}$. Therefore, the polynomials $P_{n}(\cdot ; \alpha), n=0,1, \ldots$ are called the 2-orthogonal polynomials of Laguerre type. 
3.3. A generating function. Let $G(x, t)$ be the generating function of the polynomials $P_{n}(x)=P_{n}(x ; \alpha), n \geq 0$, say

$$
G(x, t)=\sum_{n=0}^{\infty} P_{n}(x ; \alpha) \frac{t^{n}}{n !} .
$$

By using the relations (3.30), (3.31) and the definition (3.32), it is easy to verify that $G(x, t)$ satisfies the properties

$$
\begin{aligned}
(1+t) \partial_{x} G(x, t) & =t G(x, t), \\
x \partial_{x} G(x, t) & =t(1+t) \partial_{t} G(x, t)+t(t+\alpha+2) G(x, t) .
\end{aligned}
$$

Now, from (3.33) and (3.34), we easily obtain

$$
(1+t)^{2} \partial_{t} G(x, t)=[x-(1+t)(t+\alpha+2)] G(x, t),
$$

where $\partial_{x}=\partial / \partial_{x}$ and $\partial_{t}=\partial / \partial_{t}$.

Proposition 3.3. The generating function $G(x, t)$ has the form

$$
G(x, t)=e^{-t}(1+t)^{-\alpha-1} e^{x t /(1+t)} .
$$

Proof. From (3.33), we have $\partial_{x} G(x, t)=\frac{t}{1+t} G(x, t)$. By integrating both sides of this equality with respect to $x$, from 0 to $x$, we obtain

$$
G(x, t)=A(t) e^{x t /(1+t)},
$$

with $A(t)=G(0, t)$. Now, to calculate $A(t)$, substituting (3.37) in (3.34), we get (1+ t) $A^{\prime}(t)=-(t+\alpha+2) A(t)$. This yields $A(t)=C(1+t)^{-\alpha-1} e^{-t}$, where the constant $C$ is given by $C=A(0)=1$. Thus, $A(t)=(1+t)^{-\alpha-1} e^{-t}$.

REMARK 3.2. (1) The generating function obtained here has the form $G(x, t)=$ $A(t) e^{x H(t)}$, with $A(t)=(1+t)^{-\alpha-1} e^{-t}$, and $H(t)=t /(1+t)$, that is, generating function of type Scheffer. Thus, the polynomials $P_{n}(\cdot ; \alpha), n=0,1, \ldots$ appear as particular case of the class of $1 / 2$-orthogonal polynomials studied in [1].

(2) On the other hand, if we substitute $t$ by $-t$ in (3.36), we obtain

$$
e^{t}(1-t)^{-\alpha-1} e^{-x t /(1-t)}=\sum_{n=0}^{\infty}(-1)^{n} P_{n}(x ; \alpha) \frac{t^{n}}{n !}=\sum_{n=0}^{\infty} \tilde{P}_{n}(x ; \alpha) t^{n},
$$

where $\tilde{P}_{n}:=(-1)^{n} / n ! P_{n}, n=0,1, \ldots$ The polynomials $\tilde{P}_{n}(\cdot ; \alpha), n=0,1, \ldots$ are the non monic polynomials corresponding to the family of polynomials $P_{n}(\cdot ; \alpha), n=0,1, \ldots$. They are analogous to the Laguerre polynomials $L_{n}^{(\alpha)}, n=0,1, \ldots$ defined by the generating function (1.2). From the expression of the two generating functions of the polynomials $L_{n}^{(\alpha)}$ and $\tilde{P}_{n}(\cdot ; \alpha)$, we can write

$$
\sum_{n=0}^{\infty} \tilde{P}_{n}(x ; \alpha) t^{n}=e^{t} \sum_{n=0}^{\infty} L_{n}^{(\alpha)}(x) t^{n} .
$$


By using the expansion of the two members of this identity as a power series in $t$, we obtain, by identification,

$$
\begin{aligned}
& \tilde{P}_{n}(x ; \alpha)=\sum_{k=0}^{n} \frac{1}{(n-k) !} L_{k}^{(\alpha)}(x), \quad n=0,1, \ldots, \\
& P_{n}(x ; \alpha)=\sum_{k=0}^{n}(-1)^{n+k}\left(\begin{array}{l}
n \\
k
\end{array}\right) \hat{L}_{k}^{(\alpha)}(x), \quad n=0,1, \ldots
\end{aligned}
$$

\section{EXAMPLES.}

$$
\begin{aligned}
P_{0}(x ; \alpha) & =\hat{L}_{0}^{(\alpha)}(x)=1, \\
P_{1}(x ; \alpha) & =\hat{L}_{1}^{(\alpha)}(x)-\hat{L}_{0}^{(\alpha)}(x)=x-(\alpha+2), \\
P_{2}(x ; \alpha) & =\hat{L}_{2}^{(\alpha)}(x)-2 \hat{L}_{1}^{(\alpha)}(x)+\hat{L}_{0}^{(\alpha)}(x)=x^{2}-2(\alpha+3) x+(\alpha+4)(\alpha+1)+1, \\
P_{3}(x ; \alpha) & =\hat{L}_{3}^{(\alpha)}(x)-3 \hat{L}_{2}^{(\alpha)}(x)+3 \hat{L}_{1}^{(\alpha)}(x)-\hat{L}_{0}^{(\alpha)}(x) \\
& =x^{3}-3(\alpha+4) x^{2}+3\left(\alpha^{2}+7 \alpha+11\right) x-\left(\alpha^{3}+9 \alpha^{2}+23 \alpha+16\right),
\end{aligned}
$$

(3) The Languerre polynomials $\left\{L_{n}^{(\alpha)}\right\}_{n \geq 0}$ are, also, defined by the Rodrigues's formula

$$
\hat{L}_{n}^{(\alpha)}(x)=(-1)^{n} x^{-\alpha} e^{x} \frac{d^{n}}{d x^{n}}\left(x^{\alpha+n} e^{-x}\right), \quad n=0,1, \ldots
$$

Then from (3.41) we can also write

$$
P_{n}(x ; \alpha)=(-1)^{n} x^{-\alpha} e^{x} \sum_{k=0}^{n}\left(\begin{array}{l}
n \\
k
\end{array}\right) \frac{d^{k}}{d x^{k}}\left(x^{\alpha+k} e^{-x}\right), \quad n=0,1, \ldots
$$

4. Integral representations. We are, now, interested in this section in the integral representation of the linear functionals $\omega_{0}$ and $\omega_{1}$ with respect to which the sequence $\left\{P_{n}(\cdot ; \alpha)\right\}_{n \geq 0}$ is 2-orthogonal. For this, we use the same technique explained in [8]. First, we start with the 2-OPS satisfying the recurrence relation (1.11) with $\beta_{n}, \alpha_{n}$ and $\gamma_{n}$ given in (2.29). Since the sequence $\left\{P_{n}\right\}_{n \geq 0}$ is "classical", according to the characterization given in [7], we have the following theorem which is an easy application of [7, Thm. 3.1] and which we adapt to our situation:

THEOREM 4.1 [7]. For the 2-OPS $\left\{P_{n}\right\}_{n \geq 0}$ satisfying the recurrence relation (1.11) with $\beta_{n}, \alpha_{n}$ and $\gamma_{n}$ given by (2.29), its associated vector functional $\Omega={ }^{t}\left(\omega_{0}, \omega_{1}\right)$ satisfies the following vector distributional equation:

$$
D(\Phi \cup)+\Psi \mho=0,
$$

where $\Phi$ and $\Psi$ are two $2 \times 2$ polynomials matrices

$$
\Phi(x)=\left(\begin{array}{ll}
\varphi_{0}^{0}(x) & \varphi_{0}^{1}(x) \\
\varphi_{1}^{0}(x) & \varphi_{1}^{1}(x)
\end{array}\right), \quad \Psi(x)=\left(\begin{array}{cc}
0 & 1 \\
\psi(x) & \zeta
\end{array}\right),
$$


with $\varphi_{0}^{0}(x)=1, \varphi_{0}^{1}(x)=-\delta_{0}, \varphi_{1}^{0}(x)=-2 \gamma_{1}^{-1} \delta_{0}\left(x-\beta_{0}\right), \varphi_{1}^{1}(x)=1+2 \gamma_{1}^{-1} \delta_{0} \alpha_{1}$, $\psi(x)=2 \gamma_{1}^{-1}\left(x-\beta_{0}\right)$ and $\zeta=-2 \gamma_{1}^{-1} \alpha_{1}$.

Moreover, the vector functional $\tilde{\Omega}={ }^{t}\left(\tilde{\omega}_{0}, \tilde{\omega}_{1}\right)$ associated with the sequence $\left\{Q_{n}\right\}_{n \geq 0}$ is given by $\tilde{\Omega}=\Phi(x) \Omega$.

Recall that, for a linear functional $\omega$, the derivative $D \omega=\omega^{\prime}$ and the left-multiplication of $\omega$ by a polynomial $h$ are defined by $\langle D \omega, f\rangle=-\left\langle\omega, f^{\prime}\right\rangle$, and $\langle h \omega, f\rangle=$ $\langle\omega, h f\rangle, f \in \mathscr{P}$.

The main result here is

THEOREM 4.2. The two linear functionals $\omega_{0}, \omega_{1}$, associated with the 2-classical sequence $\left\{P_{n}(\cdot ; \alpha)\right\}_{n \geq 0}$, for $\alpha>-1$, respectively, have the following integral representations:

$$
\begin{aligned}
& \left\langle\omega_{0}, f\right\rangle=\int_{0}^{+\infty} \mathscr{W}_{0}(x) f(x) d x, \quad f \in \mathscr{P} ; \\
& \left\langle\omega_{1}, f\right\rangle=\int_{0}^{+\infty} \mathscr{W}_{1}(x) f(x) d x, \quad f \in \mathscr{P} ;
\end{aligned}
$$

with the weight functions $\mathcal{W}_{0}$ and $\mathcal{W}_{1}$ being given by

$$
\begin{aligned}
& W_{0}(x)=e^{-1} \varrho(x) I_{\alpha}^{*}(x) ; \\
& W_{1}(x)=e^{-1} \varrho(x)\left[x I_{\alpha+1}^{*}(x)-I_{\alpha}^{*}(x)\right],
\end{aligned}
$$

where $\varrho(x)=x^{\alpha} e^{-x}$ and $I_{\alpha}^{*}=\sum_{k=0}^{+\infty}\left(x^{k} / k ! \Gamma(k+\alpha+1)\right)$.

Proof OF THEOREM 4.1. For the proof of (4.1) and the explicit formulas for the elements of the matrix $\Psi$, see [7]. To calculate the expressions of the polynomials $\varphi_{j}^{i}$, we proceed as follows: Applying the functional $\tilde{\omega}_{0}$ (resp., $\left.\tilde{\omega}_{1}\right)$ to (2.41), since $\left\{\tilde{\omega}_{n}\right\}_{n \geq 0}$ is the dual sequence of $\left\{Q_{n}\right\}_{n \geq 0}$ we, respectively, obtain

$$
\begin{aligned}
& \left\langle\tilde{\omega}_{0}, P_{0}\right\rangle=1, \\
& \left\langle\tilde{\omega}_{0}, P_{1}\right\rangle=-\delta_{0}, \\
& \left\langle\tilde{\omega}_{0}, P_{n}\right\rangle=0 ; \quad n \geq 2,
\end{aligned}
$$

and

$$
\begin{aligned}
& \left\langle\tilde{\omega}_{1}, P_{0}\right\rangle=0, \\
& \left\langle\tilde{\omega}_{1}, P_{1}\right\rangle=1, \\
& \left\langle\tilde{\omega}_{1}, P_{2}\right\rangle=-2 \delta_{0}, \\
& \left\langle\tilde{\omega}_{1}, P_{n}\right\rangle=0 ; \quad n \geq 3,
\end{aligned}
$$

so that, by Lemma 1.1, we have $\tilde{\omega}_{0}=\lambda_{0} \omega_{0}+\lambda_{1} \omega_{1}$ and $\tilde{\omega}_{1}=\eta_{0} \omega_{0}+\eta_{1} \omega_{1}+\eta_{2} \omega_{2}$, with $\lambda_{0}=1, \lambda_{1}=-\delta_{0}, \eta_{0}=0, \eta_{1}=1$, and $\eta_{2}=-2 \delta_{0}$. Now, from [14, théorème 2.1], the functional $\omega_{2}$ can be written in terms of $\omega_{0}$ and $\omega_{1}$ as $\omega_{2}=(a x+b) \omega_{0}+c \omega_{1}$. To determine the coefficients $a, b$, and $c$, we apply $\omega_{2}$ to the polynomials $P_{0}, P_{1}$, and $P_{2}$. Using the fact that $\left\{\omega_{n}\right\}_{n \geq 0}$ is the dual sequence of $\left\{P_{n}\right\}_{n \geq 0}$, we easily obtain

$$
\begin{aligned}
& 0=\left\langle\omega_{2}, P_{0}\right\rangle=a \beta_{0}+b, \\
& 0=\left\langle\omega_{2}, P_{1}\right\rangle=a \alpha_{1}+c, \\
& 1=\left\langle\omega_{2}, P_{2}\right\rangle=a \gamma_{1},
\end{aligned}
$$


so that $a=\gamma_{1}^{-1}, b=-\gamma_{1}^{-1} \beta_{0}$ and $c=-\gamma_{1}^{-1} \alpha_{1}$. Whence, $\tilde{\omega}_{0}=\omega_{0}-\delta_{0} \omega_{1}$ and $\tilde{\omega}_{1}=$ $-2 \gamma_{1}^{-1} \delta_{0}\left(x-\beta_{0}\right) \omega_{0}+\left(1+2 \gamma_{1}^{-1} \delta_{0} \alpha_{1}\right) \omega_{1}$. Consequently,

$$
\left(\begin{array}{c}
\tilde{\omega}_{0} \\
\tilde{\omega}_{1}
\end{array}\right)=\left(\begin{array}{cc}
1 & -\delta_{0} \\
-2 \gamma_{1}^{-1} \delta_{0}\left(x-\beta_{0}\right) & 1+2 \gamma_{1}^{-1} \delta_{0} \alpha_{1}
\end{array}\right)\left(\begin{array}{c}
\omega_{0} \\
\omega_{1}
\end{array}\right)
$$

and then

$$
\Phi(x)=\left(\begin{array}{cc}
1 & -\delta_{0} \\
-2 \gamma_{1}^{-1} \delta_{0}\left(x-\beta_{0}\right) & 1+2 \gamma_{1}^{-1} \delta_{0} \alpha_{1}
\end{array}\right) .
$$

Proof of Theorem 4.2. In all the sequel, the values $\delta_{0}=-1, \beta_{0}=\alpha+2, \alpha_{1}=$ $\alpha+3$ and $\gamma_{1}=2$ are retained. Then, from Theorem 4.1 , we obtain

$$
\Phi(x)=\left(\begin{array}{cc}
1 & 1 \\
x-(\alpha+2) & -(\alpha+2)
\end{array}\right), \quad \Psi(x)=\left(\begin{array}{cc}
0 & 1 \\
x-(\alpha+2) & -(\alpha+3)
\end{array}\right) .
$$

Then, from (4.1), we obtain

$$
\begin{gathered}
\omega_{0}^{\prime}+\omega_{1}^{\prime}+\omega_{1}=0, \\
(x-\alpha-2) \omega_{0}^{\prime}-(\alpha+2) \omega_{1}^{\prime}+(x-\alpha-1) \omega_{0}-(\alpha+3) \omega_{1}=0 .
\end{gathered}
$$

From these, we easily obtain

$$
x \omega_{0}^{\prime \prime}+(2 x-\alpha+1) \omega_{0}^{\prime}+(x-\alpha) \omega_{0}=0
$$

and

$$
\omega_{1}=x \omega_{0}^{\prime}+(x-\alpha-1) \omega_{0} .
$$

To find integral representations of both the functionals $\omega_{0}$ and $\omega_{1}$, we use equations (4.13) and (4.14). The problem consists in determining weight functions $\mathscr{W}_{0}$ and $\mathscr{W}_{1}$ (depending in $\mathscr{W}_{0}$ ) such that

$$
\begin{aligned}
& \left\langle\omega_{0}, f\right\rangle=\int_{\mathscr{C}} f(x)^{\mathscr{W}} \mathcal{W}_{0}(x) d x, \quad \forall f \in \mathscr{P}, \\
& \left\langle\omega_{1}, f\right\rangle=\int_{\mathscr{C}} f(x)^{\mathscr{W}} \mathcal{W}_{1}(x) d x, \quad \forall f \in \mathscr{P} .
\end{aligned}
$$

We suppose that function $\mathcal{W}_{0}$ is regular as far as it is necessary. From (4.13), by integration by parts, we obtain

$$
\begin{aligned}
& \int_{\mathscr{C}}\left\{x^{\alpha} \mathcal{W}_{0}^{\prime \prime}(x)+(2 x-\alpha+1)^{\mathcal{W}_{0}^{\prime}}(x)+(x-\alpha)^{\mathcal{W}_{0}}(x)\right\} d x \\
& +\left[x \mathcal{W}_{0}(x) f^{\prime}(x)-\left\{(2 x-\alpha)^{\mathscr{W}} \mathcal{W}_{0}(x)+x^{\alpha} W_{0}^{\prime}(x)\right\} f(x)\right]_{\mathscr{C}}=0 .
\end{aligned}
$$

If the following condition holds:

$$
\left[x^{\mathscr{W}} \mathcal{W}_{0}(x) f^{\prime}(x)-\left\{(2 x-\alpha)^{\mathscr{W}} \mathcal{W}_{0}(x)+x^{\mathscr{C}} \mathcal{W}_{0}^{\prime}(x)\right\} f(x)\right]_{\mathscr{C}}=0,
$$


we obtain

$$
\int_{\mathscr{C}}\left\{x^{\mathscr{q}} W_{0}^{\prime \prime}(x)+(2 x-\alpha+1)^{\mathscr{q}} \mathcal{W}_{0}^{\prime}(x)+(x-\alpha)^{\mathscr{W}} \mathcal{W}_{0}(x)\right\} d x=0,
$$

which leads to

$$
x^{c} W_{0}^{\prime \prime}(x)+(2 x-\alpha+1)^{q} W_{0}^{\prime}(x)+(x-\alpha)^{q} W_{0}(x)=\lambda g(x),
$$

where $\lambda$ is an arbitrary constant and $g$ is a function representing the null functional over the path $\mathscr{b}$

$$
\int_{\mathscr{C}} g(x) x^{n} d x=0, \quad n \geq 0 .
$$

Here, we consider only the representation in terms of integrals evaluated along (an interval of) the real axis. Setting $\lambda=0$, the differential equation (4.20) becomes

$$
x^{\circ} W_{0}^{\prime \prime}(x)+(2 x-\alpha+1)^{\mathscr{W}} W_{0}^{\prime}(x)+(x-\alpha)^{\mathscr{W}} W_{0}(x)=0 .
$$

Under the transformation $\mathscr{W}_{0}(x)=e^{-x} U(s)$ with $s=2 \sqrt{x}$, the last becomes

$$
s U^{\prime \prime}(s)-(2 \alpha-1) U^{\prime}(s)-s U(s)=0 .
$$

Now, by setting $U(s)=s^{\alpha} V(s)$ in (4.23), we obtain that V satisfies the following Bessel differential equation of imaginary argument:

$$
s^{2} V^{\prime \prime}(s)+s V^{\prime}(s)-\left(\alpha^{2}+s^{2}\right) V(s)=0 .
$$

A solution of (4.24) satisfying the condition (4.18) is to be found. Thus, by choosing $\mathscr{C}=\left[0,+\infty\right.$ [, a solution of (4.24) can be taken as $V(s)=K I_{\alpha}(s)$, where $I_{\alpha}$ is the modified Bessel function of the first kind and $K$ is the normalization constant which we determine below. Then a solution of (4.22) is given by

$$
\mathcal{W}_{0}(x)=2^{\alpha} K x^{\alpha / 2} e^{-x} I_{\alpha}(2 \sqrt{x}) .
$$

The function $I_{v}(z)$, for arbitrary order $v$, is defined by (see, e.g., [13])

$$
I_{\nu}(z)=i^{-v} J_{v}(i z)=\sum_{k=0}^{+\infty} \frac{(z / 2)^{v+2 k}}{k ! \Gamma(k+v+1)}, \quad|z|<+\infty, \quad|\arg z|<\pi,
$$

where $J_{v}(z)$ is the Bessel function of order $v$. It is evident from the expansion given in the right-hand side of (4.26) that $I_{v}(z)$ is an analytic function of the complex variable $z$ and, for $z>0$ and $v \geq 0$, it is a positive function which increases monotonically as $z \longrightarrow+\infty$. The asymptotic behavior of this function as $z \longrightarrow+\infty$ is given by

$$
I_{v}(z) \sim \frac{e^{z}}{\sqrt{2 \pi z}}, \quad z \rightarrow+\infty .
$$

For small $z$, we have the asymptotic formula

$$
I_{v}(z) \sim \frac{z^{v}}{2^{v} \Gamma(v+1)}, \quad z \longrightarrow 0
$$


and, therefore, $I_{v}(0)=0$ if $v>0, I_{0}(0)=1$. Otherwise, $I_{v}$ satisfies some simple recurrence relations

$$
\begin{aligned}
2 I_{v}^{\prime}(z) & =I_{v-1}(z)+I_{v+1}(z), \\
2 v z^{-1} I_{v}(z) & =I_{v-1}(z)-I_{v+1}(z) .
\end{aligned}
$$

Now, we are ready to determine the constant $K$ and verify that the condition (4.18) holds. The normalization condition

$$
\left\langle\omega_{0}, 1\right\rangle=\int_{0}^{+\infty} \mathcal{W}_{0}(x) d x=1
$$

leads to

$$
2^{\alpha} K \int_{0}^{+\infty} x^{\alpha / 2} e^{-x} I_{\alpha}(2 \sqrt{x}) d x=1 .
$$

We first evaluate the integral

$$
\mathscr{A}=\int_{0}^{+\infty} x^{\alpha / 2} e^{-x} I_{\alpha}(2 \sqrt{x}) d x=2 \int_{0}^{+\infty} t^{\alpha+1} e^{-t^{2}} I_{\alpha}(2 t) d t
$$

But, from (4.26), we have

$$
I_{\alpha}(2 t)=\sum_{k=0}^{+\infty} \frac{t^{\alpha+2 k}}{k ! \Gamma(k+\alpha+1)} .
$$

Then

$$
\begin{aligned}
\mathscr{A} & =2 \int_{0}^{+\infty} t^{\alpha+1} e^{-t^{2}} d t \sum_{k=0}^{+\infty} \frac{t^{\alpha+2 k}}{k ! \Gamma(k+\alpha+1)} \\
& =2 \sum_{k=0}^{+\infty} \frac{1}{k ! \Gamma(k+\alpha+1)} \int_{0}^{+\infty} e^{-t^{2}} t^{2 \alpha+2 k+1} d t
\end{aligned}
$$

The exchange of integration and summation is justified by an absolute convergence argument. Next, under the transformation $u=t^{2}$, we easily obtain that

$$
\mathscr{A}=\sum_{k=0}^{+\infty} \frac{1}{k ! \Gamma(k+\alpha+1)} \int_{0}^{+\infty} e^{-u} u^{\alpha+k} d u=e .
$$

Whence, (4.31) gives $K=2^{-\alpha} e^{-1}$ and the weight function ${ }^{W_{0}}$ is given by

$$
\mathscr{W}_{0}(x)=x^{\alpha / 2} e^{-x-1} I_{\alpha}(2 \sqrt{x}) .
$$

Let us define the function $I_{\alpha}^{*}$ by

$$
I_{\alpha}^{*}(x)=x^{-\alpha / 2} I_{\alpha}(2 \sqrt{x})=\sum_{k=0}^{+\infty} \frac{x^{k}}{k ! \Gamma(k+\alpha+1)} .
$$

Thus, $\mathcal{W}_{0}$ can be written as

$$
\mathscr{W}_{0}(x)=x^{\alpha} e^{-x-1} I_{\alpha}^{*}(x)=e^{-1} \varrho(x) I_{\alpha}^{*}(x),
$$


where, $\varrho(x)=x^{\alpha} e^{x}$ is the weight function with respect to which the classical Laguerre polynomials are orthogonal. It is clear that the weight function is positive definite. Finally, we must verify that the condition (4.18) holds

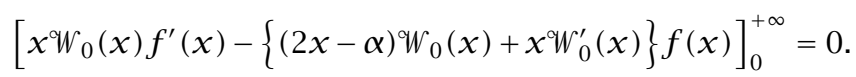

Indeed, from (4.38), we have

$$
x^{\mathcal{W}} W_{0}(x)=x^{\alpha+1} e^{-x-1} I_{\alpha}^{*}(x)=e^{-1} x I_{\alpha}^{*}(x) \varrho(x) .
$$

On the other hand, by differentiating $\mathcal{W}_{0}$, we obtain

$$
\begin{aligned}
\mathscr{W}_{0}^{\prime}(x) & =e^{-1}\left\{\varrho(x) I_{\alpha}^{* \prime}(x)+\varrho^{\prime}(x) I_{\alpha}^{*}(x)\right\} \\
& =e^{-1}\left\{\varrho(x) I_{\alpha+1}^{*}(x)+\left(\alpha x^{-1}-1\right) \varrho(x) I_{\alpha}^{*}(x)\right\} \\
& =e^{-1} \varrho(x) I_{\alpha+1}^{*}(x)+\left(\alpha x^{-1}-1\right) W_{0}(x) .
\end{aligned}
$$

Because $I_{\alpha}^{* \prime}(x)=I_{\alpha+1}^{*}(x)$ and $\varrho^{\prime}(x)=\left(\alpha x^{-1}-1\right) \varrho(x)$. Then,

$$
x^{\alpha} W_{0}^{\prime}=e^{-1} x I_{\alpha+1}^{*}(x) \varrho(x)+(\alpha-x)^{\mathscr{W}} W_{0}(x)
$$

and

$$
\begin{aligned}
(2 x-\alpha)^{\mathscr{W}} W_{0}(x)+x^{\alpha} W_{0}^{\prime}(x) & =x^{\alpha} W_{0}(x)+e^{-1} x \varrho(x) I_{\alpha+1}^{*}(x) \\
& =e^{-1} x \varrho(x)\left[I_{\alpha+1}^{*}(x)+I_{\alpha}^{*}(x)\right] .
\end{aligned}
$$

From (4.40) and (4.43), it is easy to see that at the origin when $\alpha>-1$, the condition (4.18) is verified.

Otherwise, from the asymptotic behavior (4.27) of $I_{\alpha}$, as $x \longrightarrow+\infty$, we obtain

$$
I_{\alpha}^{*}(x) \sim(4 \pi)^{-1 / 2} x^{-(2 \alpha+1) / 4} e^{2 \sqrt{x}} \text { and } I_{\alpha+1}^{*}(x) \sim(4 \pi)^{-1 / 2} x^{-(2 \alpha+3 / 4)} e^{2 \sqrt{x}} .
$$

Then,

$$
x \varrho(x) I_{\alpha}^{*}(x) \sim(4 \pi)^{-1 / 2} x^{(2 \alpha+3) / 4} e^{-x+2 \sqrt{x}}
$$

and

$$
x \varrho(x) I_{\alpha+1}^{*}(x) \sim(4 \pi)^{-1 / 2} x^{(2 \alpha+1) / 4} e^{-x+2 \sqrt{x}} .
$$

Whence, $x W_{0}(x) \longrightarrow 0$, as $x \longrightarrow+\infty$ and $(2 x-\alpha)^{\mathcal{W}_{0}}(x)+x^{\mathfrak{c} W_{0}^{\prime}}(x) \longrightarrow 0$, as $x \longrightarrow+\infty$. Then the condition (4.18) holds. Now, from (4.14), we have

$$
\begin{aligned}
\left\langle\omega_{1}, f\right\rangle & =\left\langle x \omega_{0}^{\prime}, f\right\rangle+\left\langle(x-\alpha-1) \omega_{0}, f\right\rangle \\
& =\left\langle\omega_{0}^{\prime}, x f(x)\right\rangle+\left\langle\omega_{0},(x-\alpha-1) f(x)\right\rangle \\
& =-\left\langle\omega_{0},(x f(x))^{\prime}\right\rangle+\left\langle\omega_{0},(x-\alpha-1) f(x)\right\rangle, \quad \forall f \in \mathscr{P} .
\end{aligned}
$$


By integrating by parts, we obtain

$$
\begin{aligned}
\left\langle\omega_{0},(x f(x))^{\prime}\right\rangle & =\int_{0}^{+\infty}(x f(x))^{\prime} W_{0}(x) d x \\
& \left.=x f(x)^{\mathscr{C}} W_{0}(x)\right]_{0}^{+\infty}-\int_{0}^{+\infty} x f(x)^{\mathcal{W}^{\prime}}{ }_{0}^{\prime}(x) d x .
\end{aligned}
$$

Since $\left.x f(x) \mathscr{W}_{0}(x)\right]_{0}^{+\infty}=0$ and by writing $\left\langle\omega_{1}, f\right\rangle=\int_{0}^{+\infty} f(x) W_{1}(x) d x$, we easily obtain that the weight function $W_{1}$ is given by

$$
W_{1}(x)=x^{\alpha} W_{0}^{\prime}(x)+(x-\alpha-1) W_{0}(x)=e^{-1} \varrho(x)\left[x I_{\alpha+1}^{*}(x)-I_{\alpha}^{*}(x)\right] .
$$

Note that if we take into account the dependence on the parameter $\alpha$ and setting $\mathscr{W}_{0}(x)=\mathscr{W}_{0}(x ; \alpha)$ and $\mathscr{W}_{1}(x)=\mathscr{W}_{1}(x ; \alpha)$, it may be seen that

$$
W_{1}(x ; \alpha)=W_{0}(x ; \alpha+1)-W_{0}(x ; \alpha) .
$$

\section{REFERENCES}

[1] A. Boukhemis and P. Maroni, Une caractérisation des polynômes strictement $1 / p$ orthogonaux de type Scheffer. Étude du cas $p=2$. [A characterization of strictly $1 /$-orthogonal polynomials of Scheffer type. The case $p=2$ ], J. Approx. Theory 54 (1988), no. 1, 67-91 (French). MR 89h:33013. Zbl 662.42018.

[2] C. Brezinski, Biorthogonality and its applications to numerical analysis, Monographs and Textbooks in Pure and Applied Mathematics, vol. 156, Marcel Dekker, Inc., New York, 1992. MR 92m:41001. Zbl 757.41001.

[3] C. Brezinski and M. Redivo-Zaglia, On the zeros of various kinds of orthogonal polynomials, Ann. Numer. Math. 4 (1997), no. 1-4, 67-78, The heritage of P. L. Chebyshev: a Festschrift in honor of the 70th birthday of T. J. Rivlin. CMP 97 05. Zbl 885.30005.

[4] T. S. Chihara, An introduction to orthogonal polynomials, Mathematics and its Application, vol. 13, Gordon and Breach Science Publishers, New York, 1978. MR 581979. Zbl 389.33008.

[5] K. Douak, The relation of the d-orthogonal polynomials to the Appell polynomials, J. Comput. Appl. Math. 70 (1996), no. 2, 279-295. MR 97k:33007. Zbl 863.33007.

[6] K. Douak and P. Maroni, Les polynômes orthogonaux "classiques" de dimension deux. [Two-dimensional "classical" orthogonal polynomials], Analysis 12 (1992), no. 1-2, 71-107 (French). MR 93d:33004. Zbl 767.33004.

[7] _ Une caractérisation des polynômes d-orthogonaux "classiques". [A characterization of "classical" d-orthogonal polynomials], J. Approx. Theory 82 (1995), no. 2, 177-204 (French). MR 96j:42011. Zbl 849.33004.

[8] _ On d-orthogonal Tchebychev polynomials. I, Appl. Numer. Math. 24 (1997), no. 1, 23-53. CMP 97 14. Zbl 881.33024.

[9] _ On d-orthogonal Tchebychev polynomials. II, Methods Appl. Anal. 4 (1997), no. 4, 404-429.

[10] W. Hahn, Über die Jacobischen Polynome und zwei verwandte Polynomklassen, Math. Zeit 39 (1935), 634-638. Zbl 011.06202.

[11] V. Kaliaguine, On a class of polynomials defined by two orthogonality relations, Math. Sbornik 110 (1979), 609-627, English translated in Math. USSR-Sb. 38 (1981) 563580.

[12] V. Kaliaguine and A. Ronveaux, On a system of "classical" polynomials of simultaneous orthogonality, J. Comput. Appl. Math. 67 (1996), no. 2, 207-217. MR 97f:42039. Zbl 857.42012. 
[13] N. N. Lebedev, Special functions and their applications, Prentice Hall, Inc., Englewood Cliffs, N.J., 1965, Revised English edition.Translated and edited by Richard A. Silverman. MR 30\#4988. Zbl 131.07002.

[14] P. Maroni, L'orthogonalité et les récurrences de polynômes d'ordre supérieur à deux. [Orthogonality and recurrences of polynomials of order greater than two], Ann. Fac. Sci. Toulouse Math. (5) 10 (1989), no. 1, 105-139 (French). MR 97g:42019. Zbl 707.42019.

[15] _ Two-dimensional orthogonal polynomials, their associated sets and the corecursive sets, Numer. Algorithms 3 (1992), no. 1-4, 299-311, Extrapolation and rational approximation (Puerto de la Cruz, 1992). MR 94e:42029. Zbl 779.42013.

[16] V. N. Sorokin, A generalization of a classical orthogonal polynomials and the convergence of simultaneous Padé approximants, J. Soviet Math. 45 (1989), 1461-1499.

[17] J. van Iseghem, Vector orthogonal relations. Vector QD-algorithm, J. Comput. Appl. Math. 19 (1987), no. 1, 141-150. MR 89c:65025. Zbl 626.65013.

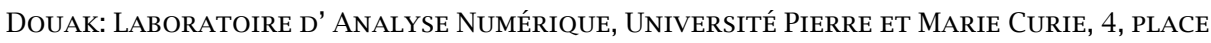
Jussieu, 75252, PARIS, CEDEX 05, FrANCE

E-mail address: douak@ann.jussieu.fr 


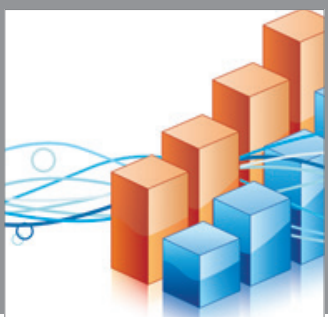

Advances in

Operations Research

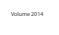

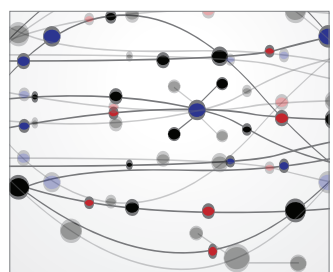

\section{The Scientific} World Journal
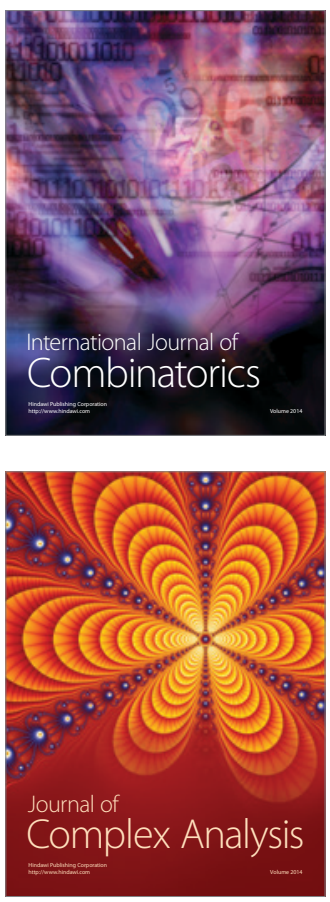

International Journal of

Mathematics and

Mathematical

Sciences
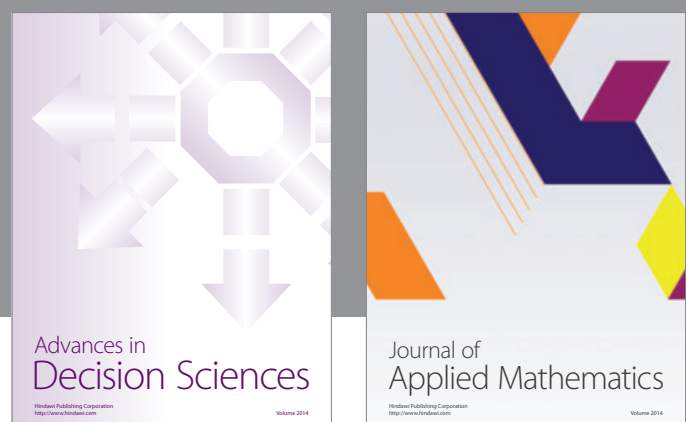

Journal of

Applied Mathematics
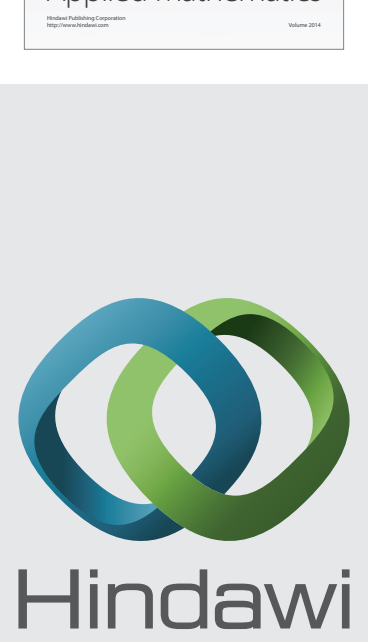

Submit your manuscripts at http://www.hindawi.com
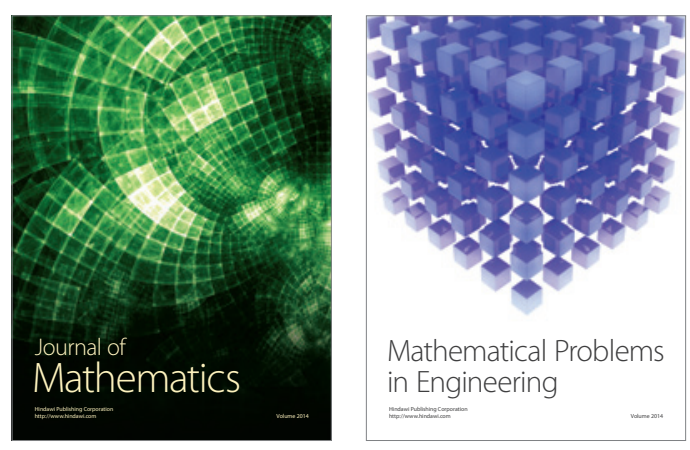

Mathematical Problems in Engineering
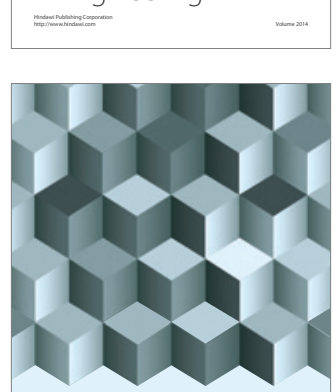

Journal of

Function Spaces
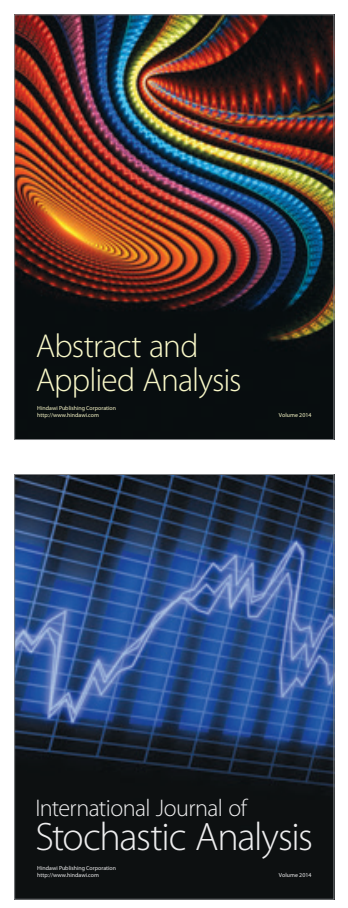

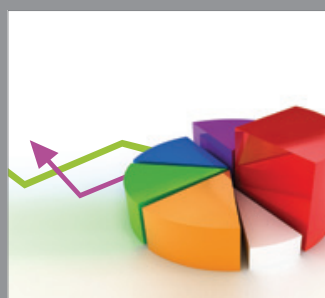

ournal of

Probability and Statistics

Promensencen
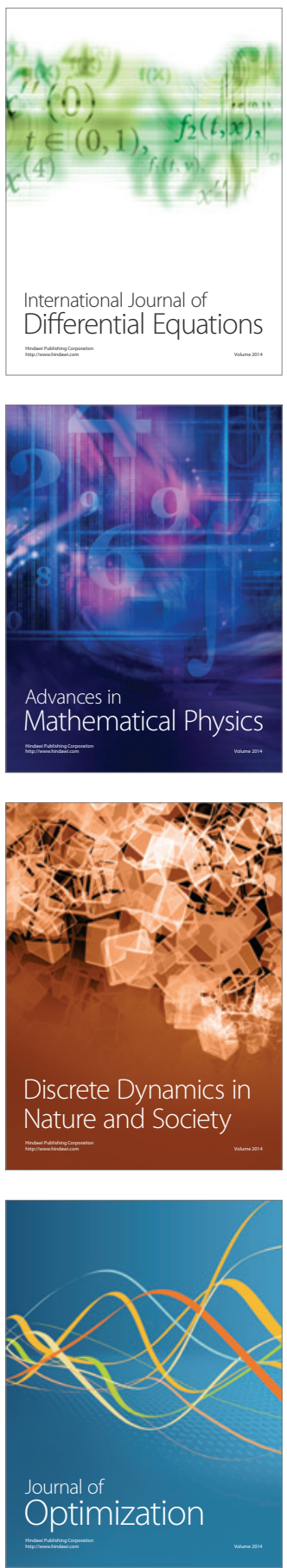